\title{
Attitude Matters! How Attitude towards Bariatric Surgery Influences the Effects of Behavioural Weight Loss Treatment
}

\author{
Kerstin Bauer ${ }^{a} \quad$ Sandra Schild ${ }^{a}$ Helene Sauer $^{a} \quad$ Martin Teufel $^{b}$ \\ Andreas Stengel $^{a, c}$ Katrin Elisabeth Giel ${ }^{a}$ Philipp Schellhorn ${ }^{d}$ Florian Junne $^{a, e}$ \\ Andreas Nieß ${ }^{d}$ Stephan Zipfel ${ }^{a}$ Isabelle Mack ${ }^{a}$ \\ aDepartment of Psychosomatic Medicine and Psychotherapy, University Hospital Tübingen, Tübingen, Germany; \\ bLVR-Clinic for Psychosomatic Medicine and Psychotherapy, University of Duisburg-Essen, Essen, Germany; \\ 'Department for Psychosomatic Medicine, Charité-Universitätsmedizin Berlin, Corporate Member of Freie Universität \\ Berlin, Humboldt-Universität zu Berlin and Berlin Institute of Health, Charité Center for Internal Medicine and \\ Dermatology, Berlin, Germany; ${ }^{d}$ Department of Sports Medicine, University Hospital Tübingen, Tübingen, Germany; \\ eDepartment of Psychosomatic Medicine and Psychotherapy, University Hospital Magdeburg, Otto von Guericke \\ University Magdeburg, Magdeburg, Germany
}

\section{Keywords}

(Behavioural) weight loss · Lifestyle $\cdot$ Motivation · Morbid obesity

\begin{abstract}
Introduction: Multidisciplinary obesity services at university hospitals usually treat patients with more complex and severe obesity. In addition, patients with Class 3 obesity, in particular, have different attitudes regarding the choices of therapy. Methods: This explorative study investigated the effect of patient attitudes towards bariatric surgery on body weight change (primary outcome) and psychological improvement (secondary outcomes: quality of life, depression, anxiety, and eating behaviour) in a 6-month moderate behavioural weight loss (BWL) programme in a university outpatient setting. Results: 297 patients with mostly Class 3 obesity participated in the programme. The patients did not yet have any indications for bariatric surgery. Of the participants, $37 \%$ had a positive attitude towards bariatric surgery (POS), whereas 38\% had a negative attitude (NEG). The drop-
\end{abstract}

karger@karger.com www.karger.com/ofa

Karger $\stackrel{\text { ' }}{5}$

GOPEN ACCESS
(C) 2021 The Author(s)

Published by S. Karger AG, Basel

This is an Open Access article licensed under the Creative Commons Attribution-NonCommercial-4.0 International License (CC BY-NC) (http://www.karger.com/Services/OpenAccessLicense), applicable to the online version of the article only. Usage and distribution for commercial purposes requires written permission. out rate was $8 \%$. NEG participants lost significantly more body weight than the POS participants (intention-to-treat population: 4.5 [SD: 6.3 ] $\mathrm{kg}$ versus 0.4 [SD: 5.8$] \mathrm{kg} ; p<0.001$ ). In both subgroups, anxiety, depression, the mental score for quality of life, and eating behaviour improved. Conclusion: A BWL treatment in a clinical setting identified 2 distinct groups with different attitudes towards bariatric surgery that were associated with different body weight change outcomes. These groups may require differently targeted programmes to achieve the best body weight loss results.

(c) 2021 The Author(s).
Published by S. Karger AG, Basel

\section{Introduction}

Obesity and its associated comorbidities, such as Type 2 diabetes, cardiovascular diseases, and orthopaedic ailments, are becoming a key, rapidly growing public health problem [1]. The risks of comorbidities increase with the degree of obesity compared to normal weight, with severe risks in Class 3 obesity [2]. As for psychological comor- 
bidities, overweight and obesity are associated with a higher prevalence of depression in adults, with subgroup differences observed. For example, the relationship between obesity and depression is higher in women [3-5]. Similarly, people with overweight or obesity are more likely to have anxiety symptoms $[6,7]$. Furthermore, obesity is associated with a higher prevalence of binge eating disorder and night eating syndrome $[8,9]$. Overall, the level of psychological distress is high in these patients, especially those with Class 3 obesity $[10,11]$.

The aims of obesity therapy are body weight loss and the reduction of comorbidities. Thus, the therapy options for obesity are behavioural weight loss (BWL) treatments or bariatric surgery $[12,13]$. Pharmacotherapy is another option for obesity management, but it is only used as an adjuvant treatment component in certain situations [13]. For BWL treatment, the body weight loss goal commonly recommended is between 5 and $10 \%$ of initial body weight within 6 months $[2,14]$. However, this is not always achieved $[15,16]$, and body weight loss maintenance remains a major challenge [17]. In contrast, the percentage body weight loss after bariatric surgery is much higher and often equivalent to $30 \%$ or more of initial body weight, even for long-term outcomes $[18,19]$. Nevertheless, psychological improvements (especially depressive symptoms) are observed with BWL treatments, irrespective of body weight loss success $[10,20]$. Similarly, depression and anxiety improve after bariatric surgery $[21,22]$. However, follow-up care is important, especially for patients with significant depression symptoms, to support body weight loss outcomes [23]. BWL treatment usually consists of a combination of nutritional, physical activity, and behavioural interventions. Consequently, a multidisciplinary approach offers the best chance for effectiveness [14].

Overall, BWL treatment programmes mainly focus on patients with Class 1 and 2 obesity [2, 24, 25]. There are no randomized controlled trials for patients with Class 3 obesity analysing the success of moderate BWL treatment programmes. Rather, the literature focuses on trials employing meal replacement strategies or extreme diets of $<1,000 \mathrm{kcal} /$ day for this patient population [16]. Pre-post design BWL treatment studies differ in intensity and duration for Class 3 obesity $[26,27]$, which may explain the high variation in body weight change outcomes across the studies [16].

According to European and American guidelines, metabolic and bariatric surgery is considered if other body weight loss attempts have failed, with a few exceptions in the case of comorbidities $[14,28]$. These evidence-based guidelines help with the decision of the ap- propriate treatment pathway, taking into account body mass index (BMI), body fat distribution, and the patient's comorbidities [29]. Patients undergoing bariatric surgery are generally younger, less well educated, have a higher BMI, and suffer more often from depression in comparison to patients participating in BWL treatments [30-32].

At the University Hospital of Tübingen, Germany, a 6-month lifestyle intervention programme for patients with obesity (VIADUKT) is offered. The programme targets patients with obesity, with the majority of them having Class 3 obesity (mean BMI $=42.7 \mathrm{~kg} / \mathrm{m}^{2}$ ). The heterogeneity of the patients taking part in VIADUKT is high, and $75 \%$ of the patients are directly referred from the multidisciplinary obesity service of the university hospital. Some patients do not fulfil the criteria for bariatric surgery due to the lack of a prior BWL treatment (BMI $\geq 40 \mathrm{~kg} / \mathrm{m}^{2}$ but $\mathrm{BMI} \leq 50 \mathrm{~kg} / \mathrm{m}^{2}$ without comorbidities), whereas others have the indication but do not wish to undergo bariatric surgery and prefer to focus on BWL treatment approaches [33]. Thus, the underlying motivations for participating in VIADUKT are quite different, and the participants have different attitudes towards bariatric surgery. Willingness to change and ambivalence towards change were identified as key variables for successful behavioural change. For example, tailoring successful interventions, identifying stages of motivation, and promoting motivation for change have been recognized as pivotal within the treatment of eating and weight disorders $[34,35]$.

In this explorative study, we address the issue that clinical outpatient BWL treatment programmes for patients with Class 3 obesity are facing in practice: a heterogeneous patient population with different attitudes towards bariatric surgery. Therefore, the aim of this study was to characterize the VIADUKT programme with regard to change of body weight as the primary outcome and quality of life, psychological factors (depression and anxiety), and eating behaviour as secondary outcomes, as well as to distinguish between patients with different attitudes towards bariatric surgery. Specifically, the focus was on patients with a positive attitude towards bariatric surgery (POS group) in contrast to those patients with a negative attitude (NEG group). We hypothesized that (i) the baseline characteristics of NEG participants, in contrast to POS participants, have lower values on measures of body weight and psychological scores; (ii) body weight loss is lower in POS participants in comparison with NEG participants; (iii) eating behaviour improves, but inferiorly, in POS vs. NEG participants; and (iv) quality of life, anxiety, and depression improve over the course of treatment in all participants. 


\section{Materials and Methods}

\section{Study Design and Participants}

This is a prospective follow-up study, recruiting participants undergoing the BWL treatment VIADUKT at the University Hospital Tübingen, Germany. The study is approved by the Ethics Committee of the University Hospital Tübingen, Germany with the number 391/2019BO2.

Recruitment was conducted via the multidisciplinary obesity service of the university hospital, consisting of an interdisciplinary team from psychosomatic medicine, endocrinology, nutritional medicine, sports medicine, and visceral surgery. This multidisciplinary obesity service is the initial contact point for bariatric surgery at the university hospital, which is significantly involved in the allocation of the therapy pathway. In addition, leaflets were distributed among general practices in the area of Tübingen and the university hospital itself.

Inclusion criteria were an age of at least 18 years; in addition, a $\mathrm{BMI}$ of at least 30 was desired but not required. Participants were excluded in the case of language difficulties. This was determined if communication via telephone was not possible.

Data from May 2014 (programme start) to September 2019 were analysed which corresponded to 297 patients having participated in the programme. Baseline characteristics were collected before intervention and included body weight, body height, and questionnaires for quality of life, anxiety, depression, and eating behaviour. The details for these measurements are described in detail in the section "outcomes."

\section{Treatment}

"VIADUKT" is an acronym for "Verhaltensintervention bei Adipositas am UKT," which is a BWL treatment for patients with obesity at the University Hospital Tübingen. The intervention consists of ten 75-minute group meetings and twenty 45-min guided exercise sessions delivered by a multidisciplinary team, which consisted of the disciplines psychosomatic medicine, nutritional science, and sports medicine. The group meetings focus on nutritional education and promote lifestyle changes. Specifically, patients are educated on motivational strategies, flexible and controlled eating patterns, basics for regular physical activity, stress management techniques, and strategies for long-lasting weight loss maintenance. This is according to the German clinical practice guidelines for obesity [36].

Demographics were assessed by standard questionnaires ahead of the programme, including nationality as an answering option using a blank field. Body weight was assessed using a calibrated scale at the end of or before the group meetings. Criteria for successful intervention exposure were participation of at least $80 \%$ in the group meetings and exercise sessions; otherwise, the participants were defined as non-completers. Prior to the course, the participants contributed to the costs with $20 \%$ of the total amount; the rest of the amount was covered by health insurance. In case of an $80 \%$ participation in the programme, the personal contribution was refunded by the health insurance, independent of body weight change outcome.

\section{Outcomes}

Body weight change in kilogram was the primary outcome for this programme and was measured by standard techniques, using the same calibrated scale (Seca 701; Vogel \& Halke, Hamburg, Ger- many) before (T0) and at the end of the intervention (6 months later, T1). For the measurement, participants wore lightweight clothes and no shoes. Height was measured in centimetres using a stadiometer at baseline only.

Secondary outcomes were measured via questionnaires for quality of life, depression, anxiety, and eating behaviour and were also assessed at T0 and T1. The applied questionnaires are described below in detail. The patients were asked once about their attitude towards bariatric surgery using the open question: "What is your attitude towards bariatric surgery?" The answers were categorized into: "Yes, bariatric surgery is an option for me" (POS), "No, bariatric surgery is not an option for me" (NEG), "I am not sure" (Uncertain), and if no information was given, the participants were referred to as "Not Clear". The results for primary and secondary outcomes are presented for the whole group and for the 2 subgroups POS versus NEG.

The Patient Health Questionnaire (PHQ-9) is a screening tool for depression and consists of 9 items. The possible answer options are "not at all," "several days," "more than half of the days," or "nearly every day." Once completed, a score is calculated and categorized into none to minimal (0-4), mild (5-9), moderate (10$14)$, moderately severe (15-19), or severe (20-27) levels of depression [37].

The Generalized Anxiety Disorder Questionnaire (GAD-7) is used for screening generalized anxiety disorder and consists of 7 items. The possible answer options are "not at all," "several days," "more than half of the days," or "nearly every day." Once completed, a score is calculated and categorized into minimal (0-4), mild (5-9), moderate (10-14), or severe (15-21) anxiety [38].

The Short Form 12 contains 12 items to assess health-related quality of life, consisting of mental and physical component summaries that compare the patient's outcome to the general US population [39]. The average is set at 50, with a standard deviation (SD) of 10 and higher (lower) scores indicate a better (worse) health status.

The German version of the Three-Factor Eating Questionnaire, which contains 3 subscales ("Cognitive Restraint," "Disinhibition," and "Feelings of Hunger"), focuses on eating behaviour and largely consists of dichotomous yes/no questions [40].

The above questionnaires have been used and validated in patients with obesity (anxiety and depression [22, 41], quality of life $[42,43]$, and eating behaviour $[22,44,45])$.

\section{Statistical Analysis}

The data analysis was performed with SPSS Statistics for Windows, Version 24.0 [46]. For continuous variables, normal distribution was tested with the Kolmogorov-Smirnov test and equality of variances between groups with Levene's test. Data are reported as mean (SD), confidence interval along with the median (interquartile range) due to non-parametric data distribution. Frequencies are given as percentages (\%).

Baseline differences between the 2 subgroups POS and NEG were tested with the Mann-Whitney $U$ test for metrical data and with the $\chi^{2}$ test or the Fisher's exact test for nominal data when the frequency of cells was too low. Differences between T0 and T1 for the whole study population (body weight and psychometry) were analysed with the Wilcoxon signed-rank test. To analyse group (POS vs. NEG) and time (T0 vs. T1) interactions, the robust $2 \times 2$ ANOVA was conducted along with the non-parametric van der Waerden test for data confirmation [47]. Since the results did not 
deviate between the ANOVA and van der Waerden tests, only the ANOVA data are reported. In case of different baseline levels between the NEG and POS groups, an ANCOVA (dependent variable: differences in scores between T1 and T0, fixed factor: POSNEG group, covariable: score at T0) was performed. Two-tailed tests were applied throughout the manuscript. To account for multiple testing, only $p$ values $<0.001$ were considered as statistically significant and $p$ values $<0.05$ as a trend. The corresponding effect sizes for the $\chi^{2}$ test and Mann-Whitney $U$ test are defined as follows: $0.1=$ low effect, $0.3=$ medium effect, and $0.5=$ high effect.

For the primary outcome, the results for the total group and both subgroups were presented in online suppl. Table 1 (see www. karger.com/doi/10.1159/000517850 for all online suppl. material) separately for the responders (weight reduction achieved) and the non-responders (weight reduction not achieved or weight gain occurred). Response was further differentiated into low (0-4.9\%), medium (5-9.9\%), and high ( $\geq 10 \%)$ percentage body weight loss.

For secondary outcomes, "minimal clinically important differences" (MCIDs) were analysed when possible. For the analysis, MCIDs were set for anxiety and depression at a value of 4 points $[37,48]$ and for quality of life at 3 points [49]. Additionally, for anxiety and depression, the scores were categorized into subgroups according to the manual guidelines (depression: $0-4=$ minimal, $5-9=$ mild, $10-14=$ moderate, $15-21=$ severe depres sion; anxiety: $0-4=$ minimal, $5-9=$ mild, $10-14=$ moderate, $15-21$ $=$ severe anxiety) and the percentages of participants who switched subgroups (improved or worsened) or remained stable in their subgroup.

A hypothesis-driven simple linear regression was calculated to predict body weight change (delta body weight in kilogram between $\mathrm{T} 0$ and $\mathrm{T} 1$ ) based on the attitude towards bariatric surgery POS or NEG.

\section{Missing Data Imputation}

Missing data for primary and secondary outcomes were replaced by the predictive mean matching method after data were analysed with Little's test of missing completely at random to detect whether the missing data were random [50].

Primary Outcome

For the per-protocol population, participants were only included if body weight $(\mathrm{kg})$ was provided at $\mathrm{T} 0$ and $\mathrm{T} 1$ of intervention, and if they were not classified as a non-completer ( $\geq 80 \%$ exposure). The per-protocol population consisted of 267 participants (missing data at T0 and T1: $n=7$; non-completer: $n=23$ ).

The intention-to-treat population consisted of 297 participants. The results of the predictive mean matching method did not substantially deviate from the last observation carried forward method, the latter having been methodologically criticized [51] but used by most of the lifestyle intervention studies cited in this article.

\section{Secondary Outcome}

In case of missing data for quality of life, depression, anxiety, or eating behaviour, either for single items or complete questionnaires at either T0 or T1, a multiple imputation with 5 iterations was performed $[48,49]$. Cases with single-item imputations were included in the per-protocol population, and cases with complete questionnaires and/or single-item imputations were included in the full-data population. Participants were excluded if secondary outcome data were missing at both time points, T0 and T1 $(n=19)$.
For the imputations, the predictive mean matching method with the predictors sex and age was used to replace single items and/or complete questionnaires that were missing at T0 or T1 [52]. The percentage of missing data for complete questionnaires ranged between 5 and $21 \%$ for quality of life, depression, anxiety, or eating behaviour at T0 or T1 (T0 eating behaviour: 5.5\%, T1 eating behaviour: $20.1 \%$, T0 anxiety: $11.9 \%$, T1 anxiety $20.8 \%$, T0 depression $18.3 \%$, T1 depression $20.8 \%$, T0 quality of life $7.2 \%$ and T1 quality of life $20.8 \%$ ).

\section{Results}

A total of $297\left(\right.$ mean BMI $\left.=42.7 \mathrm{~kg} / \mathrm{m}^{2}\right)$ patients took part in the VIADUKT lifestyle intervention programme at a university hospital setting. Out of these, 23 participated in $<80 \%$ of the meetings and were classified as noncompleters. Thus, the drop-out rate was $8 \%$ for this programme.

From the total sample, $113(38 \%)$ participants declared to not desire bariatric surgery (NEG), whereas $111(37 \%)$ participants wanted to receive bariatric surgery (POS). Thirty participants were undecided (Uncertain), and for 43 participants, no information about their decision was available (Not Clear). In this study, the focus is on the differences between the NEG and POS groups, which represent $75 \%$ of the study population.

\section{Baseline Characteristics}

The POS group scored significantly higher in depression, lower in quality of life (mental and physical scores), and were younger $(p<0.001)$. In addition, POS participants tended to be less well educated, had higher scores in anxiety and lower scores in feelings of hunger, and were more often foreign $(p<0.05)$.

In contrast, body weight, sex, personal status, composition of household, and the 2 subscales of eating behaviour "cognitive restraint" and "disinhibition" were similar between the groups. A detailed overview of the baseline characteristics for the total group and the subgroups is presented in Table 1.

\section{Characteristics of Non-Completers}

The participants who did not complete the programme $(n=23)$ had a mean age of 42.4 (SD: 12.3$)$ years, a body weight of 127.6 (SD: 23.3) $\mathrm{kg}$ and a BMI of 44 (SD: 5.7) $\mathrm{kg} / \mathrm{m}^{2}$, and $73.9 \%$ were women. The drop-outs consisted of $35 \%$ NEG, $17 \%$ POS, and $48 \%$ Not Clear. There were no significant baseline differences between programme completers and non-completers (drop-outs). 


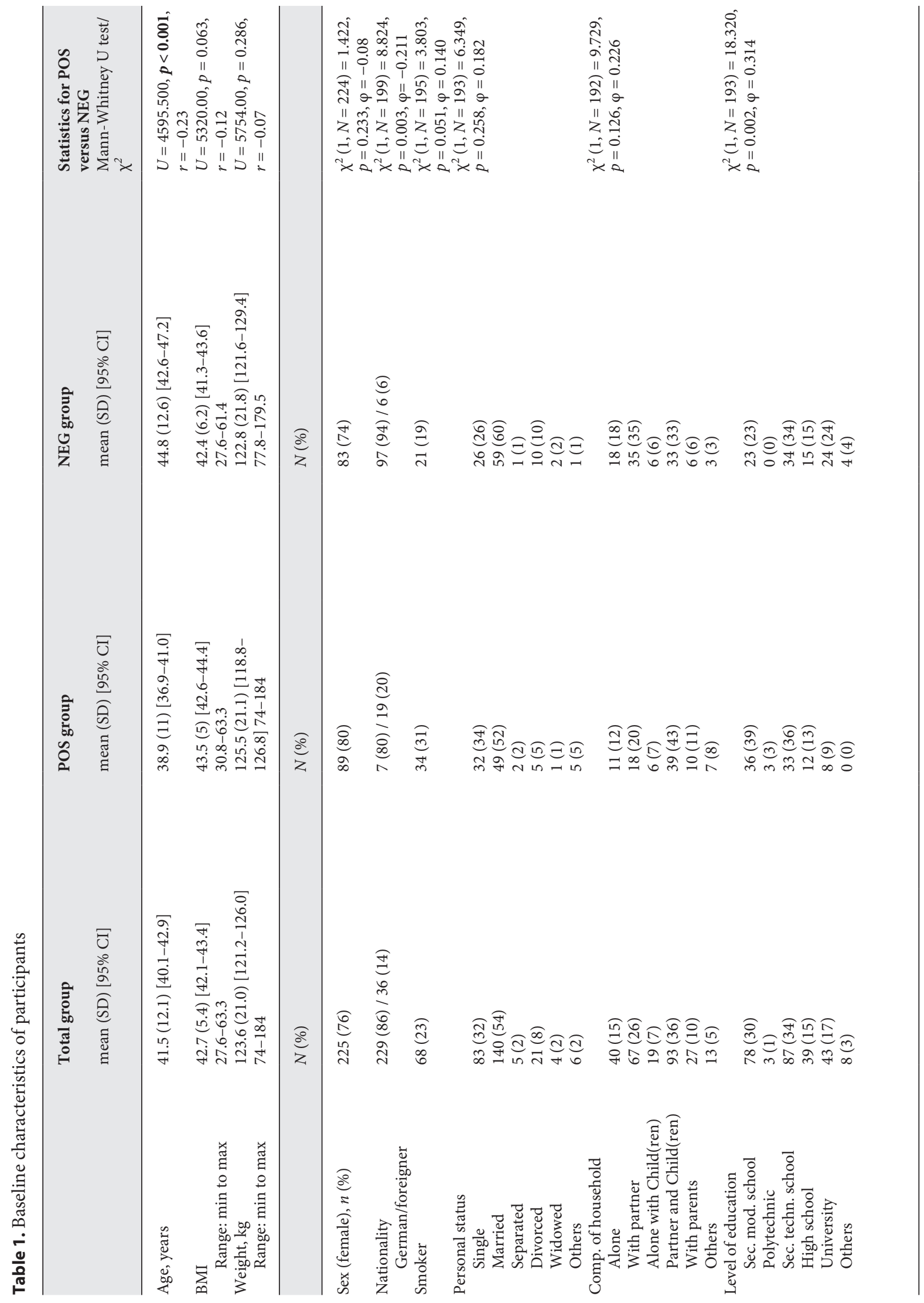




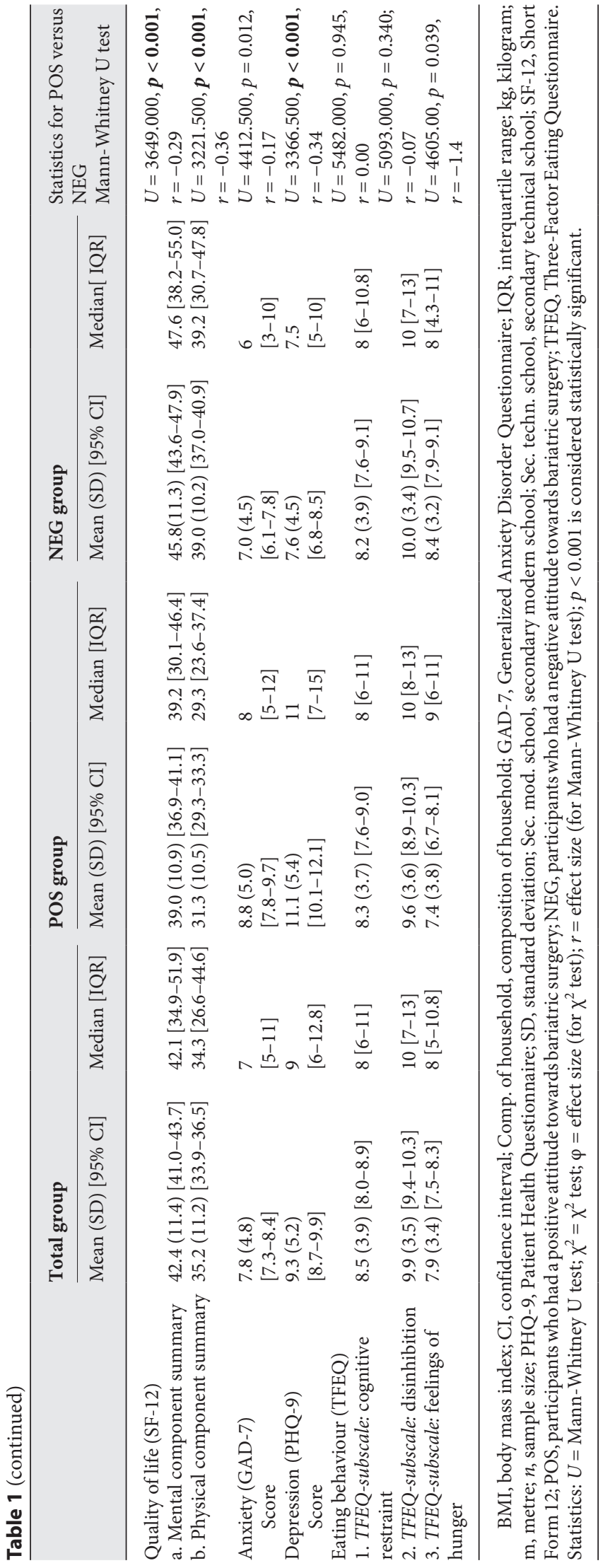

\section{Body Weight Change}

A detailed overview of the body weight change data is presented in online suppl. Table 1.

\section{Per-Protocol Population}

The mean percentage reduction of body weight was $2 \%$. This equates to a mean body weight change of -2.4 (SD: 6.1$) \mathrm{kg}$, ranging from $-28.2 \mathrm{~kg}$ to $+15 \mathrm{~kg}(z=-6.325$, $p<0.001, n=267, r=-0.39$ ). Mean percentage reduction of body weight was $4 \%$ in the NEG group and $0.4 \%$ in the POS group, equating to a mean body weight change of -4.5 (SD: 6.3 ) $\mathrm{kg}$ and -0.6 (SD: 5.8 ) kg, respectively (F $[1,207]=22.895, p<0.001$, partial $\eta^{2}=0.1$ ).

\section{Intention-to-Treat Population}

The mean percentage reduction of body weight for the total group was $2 \%$, which equates to a mean body weight change of -2.4 (SD: 6.1) $\mathrm{kg}(z=-6.309, p<0.001, n=297$, $r=-0.37)$. In comparison, the mean percentage reduction of body weight was $4 \%$ for the NEG group $(n=113)$ and $0.3 \%$ for the POS group $(n=111)$, equating to a mean body weight change of -4.5 (SD: 6.3 ) $\mathrm{kg}$ and -0.4 (SD: $5.8) \mathrm{kg}$, respectively $(\mathrm{F}[1,222]=26.600, p<0.001$, partial $\left.\eta^{2}=0.107\right)$.

\section{Questionnaires}

The data were analysed for the per-protocol population and for the full-data analysis. Since no differences between the approaches were found, the results of the full-data analysis are presented.

Quality of Life: Short Form 12 Questionnaire (SF12)

Overall, the physical and mental component summaries improved significantly (Fig. 1). Similar patterns were also found within the 2 subgroups POS and NEG with one exception: For the POS group, the physical component summary did not change over the course of time (Fig. 2).

Furthermore, the results were scored based on MCIDs (online suppl. Table 2). For the mental and physical scores, the value was set at 3 points [49]. In total, the physical score improved in $41 \%$, remained stable in $36 \%$, and worsened in $23 \%$ of the patients; the mental score improved in $46 \%$, remained similar in $27 \%$, and worsened in $27 \%$ of the patients. In detail, for the POS and NEG groups, half of the participants achieved MCID for the mental scores, as well as $50 \%$ of the NEG group for the physical scores. In contrast, only onethird of the POS group reached the MCID for the physical scores. The results are reported in detail in online suppl. Table 2. 
Fig. 1. Secondary outcomes of the whole study population: quality of life, depression, anxiety, and eating behaviour of the total study population for pre- (T0) and post-intervention (T1): Scores for physical quality of life (A.1), mental quality of life (A.2), depressive symptoms (B), anxiety symptoms (C), and eating behaviour (D) are presented. The data are shown as boxwhiskers (median with upper and lower quartiles), whose difference describes the interquartile range (IQR) and minimum and maximum (=whiskers). Mean is depicted as “+." Increases from T0 to T1 for quality of life and cognitive restraint, as well as decreases from T0 to T1 for anxiety, depression, disinhibition, and feelings of hunger, represent an improvement. $* * *$ Significant differences between T0 and T1 $(p<0.001)$.

\section{A.1. Quality of Life: Physical Component}

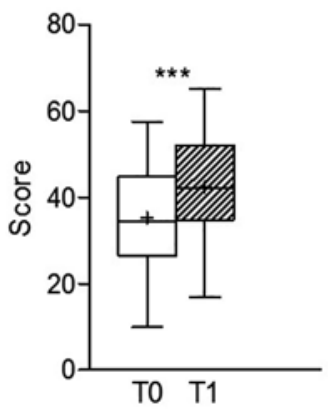

C. Anxiety

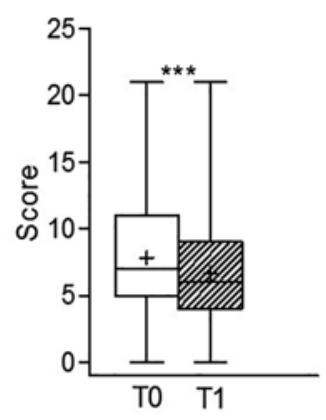

A.2. Quality of Life: Mental Component

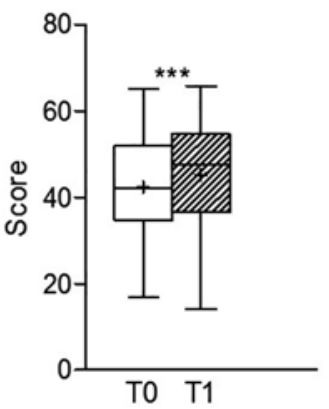

B. Depression

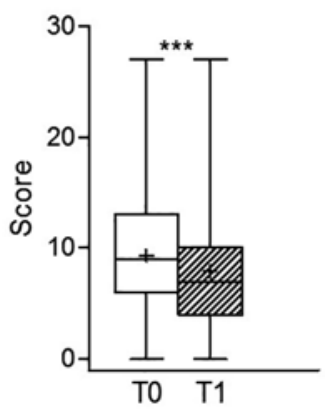

\section{Eating Behaviour}

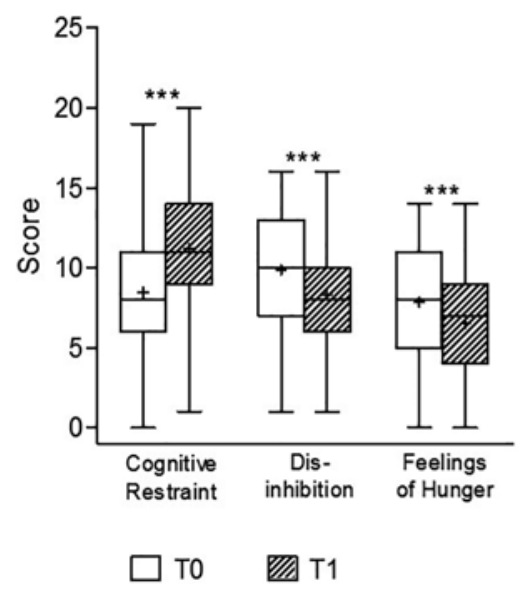

Anxiety and Depression: Generalized Anxiety

Disorder Questionnaire (GAD-7) and Patient Health Questionnaire (PHQ-9)

Anxiety and depression symptoms improved (Fig. 1), and both subgroups (POS and NEG) benefited equally when analysed separately (Fig. 2). The values for depression improved in $38 \%$, worsened in $17 \%$, and remained stable in $45 \%$ of the patients. For anxiety, the values improved in $33 \%$, worsened in $13 \%$, and remained stable in $54 \%$ of the patients.

In addition, the MCID was calculated conservatively, setting the boundary value at 4 points for both scores $[37,48]$. For anxiety, MCID improved in $25 \%$, worsened in $8 \%$, and remained similar in $67 \%$ of the patients. Likewise, for depression, the MCID improved in 29\%, worsened in $6 \%$, and remained stable in $65 \%$ of the patients. The results for each subgroup separately are comparable to these findings and are reported in online suppl. Table 2.

Eating Behaviour: Three-Factor Eating Questionnaire (TFEQ)

The subscales "disinhibition" and "feelings of hunger" decreased from pre- to post-treatment. Although "cognitive restraint" increased in the total group (Fig. 1), the patterns were similar between the POS and NEG groups (Fig. 2).

\section{Prediction of Body Weight Loss}

A hypothesis-driven simple linear regression was considered to predict body weight change $(\mathrm{kg})$ based on the attitude towards bariatric surgery POS or NEG. Attitude towards bariatric surgery was able to significantly predict body weight loss $(\mathrm{F}[1,222]=26.6, p<0.001)$. The $R^{2}$ for 


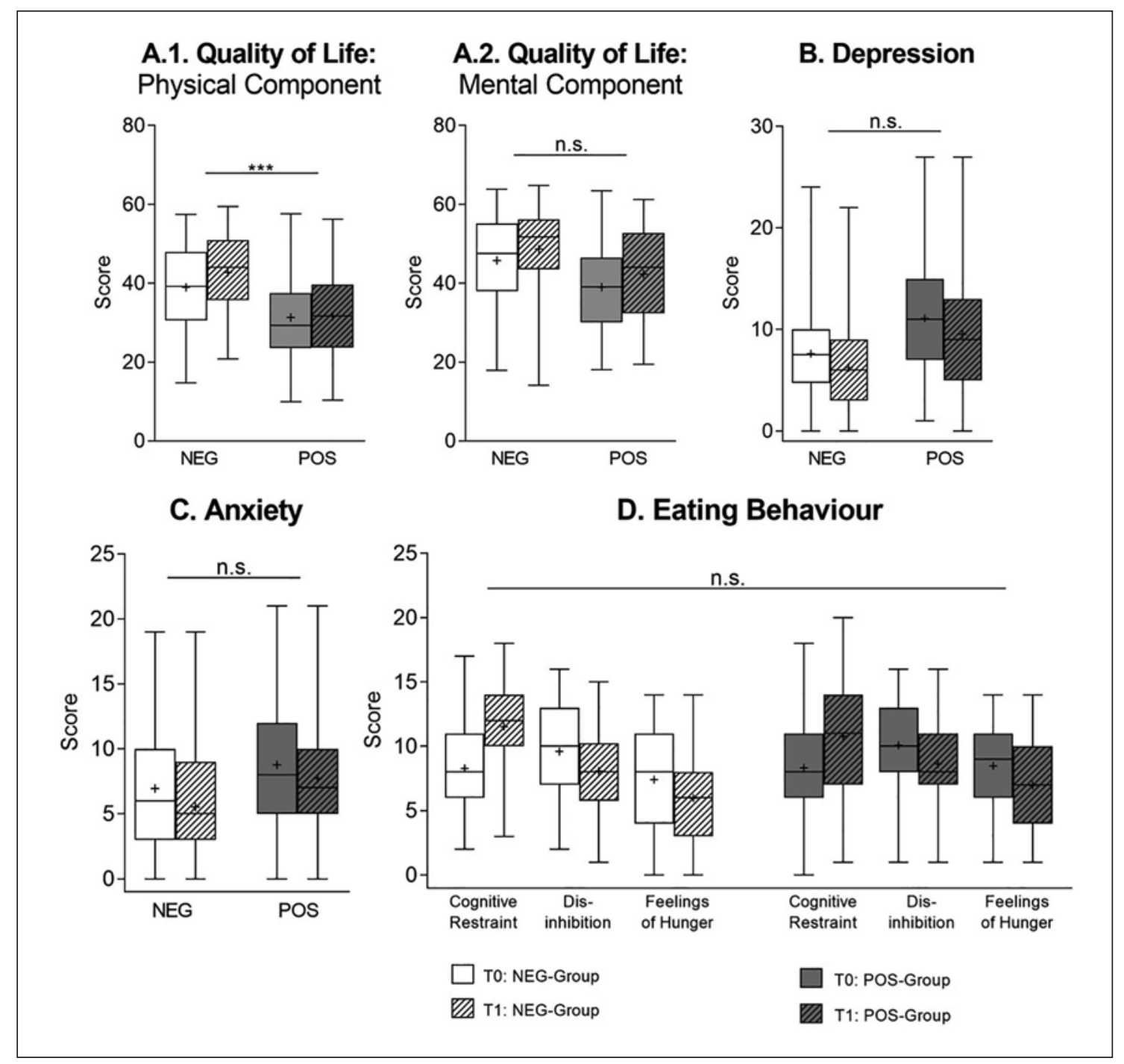

Fig. 2. Secondary outcomes of POS versus NEG: quality of life, depression, anxiety, and eating behaviour in patients with POS and NEG for pre- (T0) and post-intervention (T1): Scores for physical quality of life (A.1), mental quality of life (A.2), depressive symptoms (B), anxiety symptoms (C), and eating behaviour (D) are presented. The data are shown as box-whiskers (median with upper and lower quartiles), whose difference describes the interquartile range (IQR) and minimum and maximum (=whiskers). Mean is depicted as “+." Increases from T0 to T1 for quality of life and cognitive restraint, as well as decreases from T0 to T1 for anxiety, depression, disinhibition, and feelings of hunger, represent an improvement. Statistics for time $\times$ group interactions are indicated: $* * *$ Significant difference $(p<0.001)$; n.s., not significant. POS, patients with a positive attitude towards bariatric surgery; NEG, patients with a negative attitude towards bariatric surgery.

\section{Discussion}

This is the first study assessing exploratively the impact of patient attitudes towards bariatric surgery in the context of a BWL treatment for patients with severe obesity seeking support at a university hospital setting. As hypothesized (i), baseline characteristics indicated differences between participants of the NEG and POS groups. 
Overall, the POS group strongly resembled baseline characteristics reported for patients before bariatric surgery [30-32], with POS participants being younger and having a higher depression score with a lower score in quality of life. The POS participants also tended to be less well educated and were more likely to be foreign and have a higher smoking rate. An inverse correlation between the level of education and smoking has been previously identified [54]. Thus, it appears as though socioeconomic status plays a role in the willingness to consider bariatric surgery as an option in this cohort.

As hypothesized (ii), body weight loss in the POS group was lower in comparison to the NEG group. A BWL treatment similar to the presented study here but conducted in an outpatient, non-hospital-associated setting is depicted by Rudolph et al. [55]: 190 participants with a mean BMI of 44.1 (SD: 6.2$) \mathrm{kg} / \mathrm{m}^{2}$ had a mean body weight change of $-4.5 \mathrm{~kg}$ in 1 year. Due to the non-hospital-based setting, it can be expected that the ratio of patients with a positive attitude towards bariatric surgery may be low, but this is mere speculation.

Overall, the range of body weight change achieved in BWL treatments in patients with Class 3 obesity is broad across and within studies, and the reasons, especially for the latter, are unclear [16]. None of the studies reported on the attitude towards bariatric surgery, which could be an explaining factor for the high body weight change variability within them, similar to our findings. Since selfmotivation is a decisive factor for body weight change [56, 57], this raises the question of whether a relationship exists between not desiring bariatric surgery and self-motivation for behaviour change. However, we are unable to determine this from the results of the study, and further investigations are necessary.

Eating behaviour improved as described in hypothesis (iii), but against our assumptions, no differences were observed between the POS and NEG participants, despite distinct body weight change differences. We assume that participants of the POS group started reflecting on their dietary intake and eating behaviour but lacked implementation. This may have led to an overestimation of their improvements in eating behaviour. Overall, it is well documented that eating behaviour and dietary intake patterns improve during BWL treatments, and that disinhibition and feelings of hunger do not directly correlate with body weight change $[58,59]$.

In line with hypothesis (iv), the participants benefited with regard to psychological aspects. The MCIDs for anxiety and depression improved for $26-32 \%$ of the patients and were unchanged for $62-67 \%$. For quality of

Surgery Attitude and Behavioural Weight

Loss life, the MCID was reached by almost $50 \%$ in the mental score. Differences in the physical scores were observed, with $50 \%$ MCID in the NEG group and over 30\% in the POS group. Thus, despite the blunted weight loss of the POS group, with $44 \%$ not achieving body weight change during the VIADUKT programme (non-responders), the intervention affected the physical quality of life of almost one-third of the group. The participants whose quality of life improved achieved a body weight change of -3.5 (SD: 5.3 ) kg, whereas the others lost 1.5 (SD: 6.2) $\mathrm{kg}$. This trend of the greater the body weight loss, the more likely the change in quality of life was also reported by Lasikiewicz et al. [20] and Kolotkin et al. [60]. However, the change in depression is not as highly associated with body weight change [20] and can be independent of it [10].

Considering that the baseline characteristics of the POS participants are similar to patients who underwent a bariatric surgery, and that the BWL treatment outcomes are rather poor, it could be argued whether this treatment is ideal for this particular subpopulation. It should also be questioned very critically in light of the current ongoing debate as to whether or not conservative weight management programmes should be the first treatment option in individuals with a BMI $\geq 35 \mathrm{~kg} / \mathrm{m}^{2}$, since surgical procedures have turned out to be highly effective and safe, even for lower obesity classes [19]. Nevertheless, 6 months of working towards improving lifestyle factors resulted in improved eating behaviour and mental strength. This might have an impact on long-term weight regulation, and patients may also continue to benefit from the new skills after bariatric surgery, but this is speculation as the literature on this issue is contradictory $[61,62]$. In addition, group dynamics are known to be an important factor for body weight change in BWL treatments [63]. Having POS and NEG participants in the same group setting appears to be difficult with regard to goals, and peer support can hardly be expected.

Another perspective on the results could include motivational issues. Although the relationship between attitude towards bariatric surgery and motivation to change is not clear, participants might benefit more from the behavioural intervention if their motivational stage was identified. This information could be utilized to better tailor behavioural interventions to the individual and provide an opportunity to work on their motivation and ambivalence towards change, for example, by applying techniques such as motivational interviewing. This technique has been shown to also work well in other serious and chronic conditions $[34,35]$.

Obes Facts 2021;14:531-542 539 
The presented study has several strengths and limitations. First of all, one strength is that we assessed the attitude towards bariatric surgery to allow a stratified analysis for a reasonable sample size at baseline and over the course of the intervention. Various facets of psychological well-being were investigated, which are often neglected in the evaluation of such programmes. In addition, the drop-out rate was very low at $8 \%$, which may have contributed to the fact that the costs of the programme were only fully covered by health insurance in the case of $80 \%$ programme participation of the individual participant. A particular strength of this study is that it illustrates the challenges that clinics are facing when offering outpatient weight loss programmes that are directly connected to a multidisciplinary obesity service. However, this is also a limitation, as the results of this study cannot be transferred to the general population of obese patients, as the target group addressed here is highly burdened and has a more complex health condition and medical history. A limitation is the uncontrolled study design of this investigation. As outlined above, we did not find a single randomized controlled trial comparing moderate BWL treatments in patients with Class 3 obesity [16]. All other studies dealing with this topic had a similar pre- and post-design. Furthermore, we did no follow-up to analyse weight loss maintenance. In general, weight loss maintenance is problematic and requires a continuous multidisciplinary approach [17].

Finally, alongside other studies dealing with BWL treatment procedures, we showed that this method has its limitations as discussed in detail elsewhere [16]. Nevertheless, not all patients wish to undergo either bariatric surgery or a meal replacement/extreme energy restriction diet. For these patients or still undecided patients, it is extremely important to offer BWL treatments with moderate caloric restrictions in order to support the stabilization and/or improvement of body weight, reduce severity of comorbidities, and improve quality of life and psychological health. Without intervention, further body weight gain and aggravation of comorbidities are likely. Thus, providing options for moderate BWL treatments for patients with Class 2 or 3 obesity should not be undervalued, as they may provide an important interim step to assist decision making and stabilize physiological and psychological factors. The decision for or against treatment options should be based on the personal situation and desires of the patient.

\section{Conclusions}

In summary, attitude towards bariatric surgery was a predictor for body weight change. However, desirable group dynamics might be hindered if underlying attitudes and goals are too heterogeneous between group members. Therefore, we recommend assessing the attitudes towards bariatric surgery for participants of BWL treatment groups. This information could then ideally be used by group facilitators to tailor sessions and topics covered. Furthermore, education delivery techniques, support, and mitigation of group dynamics as well as leading conversations should be adapted. Special rules could then help to keep the focus on the BWL treatment. For example, while bariatric surgery should not be a topic during the sessions, it could be addressed individually or at a special group meeting with those who wish to have the procedure.

\section{Acknowledgments}

We thank Jessica Cook for her contribution in editing and Dr. Nazar Mazurak for discussion. Furthermore, we thank the study participants for their contribution to the study.

\section{Statement of Ethics}

The study is approved by the Ethics Committee of the University Hospital Tübingen, Germany, with the number 391/2019BO2. The participants gave their written informed consent.

\section{Conflict of Interest Statement}

The authors have no conflicts of interest to declare.

\section{Funding Sources}

The authors acknowledge support by the Deutsche Forschungsgemeinschaft and the Open Access Publishing Fund of Tübingen University. I.M. received a grant from the Ministry of Science Baden-Württemberg and the European Social Fund.

\section{Author Contributions}

I.M., M.T., and H.S. contributed to conceptualization; K.B., I.M., M.T., and K.G.E. contributed to methodology; K.B. and I.M. contributed to software; K.B., H.S., S.S., and P.S. contributed to validation; K.B. and I.M. contributed to formal analysis; S.S., H.S., P.S., and K.B. contributed to investigation; S.Z. and A.N. contrib- 
uted to resources; K.B. and I.M. contributed to data curation; K.B. and I.M. contributed to writing - original draft preparation; all authors contributed to writing - review and editing; K.B. and I.M. contributed to visualization; I.M., M.T., and A.S. contributed to supervision; M.T., S.Z., A.N., A.S., I.M., H.S., S.S., and P.S. contributed to project administration; S.Z. and I.M. contributed to funding acquisition. All authors have read and agreed to the published version of the manuscript.

\section{Data Availability Statement}

All data generated or analysed during this study are included in this article and/or its online suppl. material files. Further enquiries can be directed to the corresponding author.

\section{References}

1 Apovian CM. Obesity: definition, comorbidities, causes, and burden. Am J Manag Care. 2016;22(7 Suppl):s176-85.

2 World Health Organization. Obesity: preventing and managing the global epidemic. Report of a WHO consultation. World Health Organ Tech Rep Ser. 2000;894:i-xii, 1-253.

3 Fabricatore AN, Wadden TA. Psychological aspects of obesity. Clin Dermatol. 2004 JulAug;22(4):332-7.

4 Luppino FS, de Wit LM, Bouvy PF, Stijnen T, Cuijpers P, Penninx BW, et al. Overweight, obesity, and depression: a systematic review and meta-analysis of longitudinal studies. Arch Gen Psychiatry. 2010;67(3):220-9.

5 Pereira-Miranda E, Costa PRF, Queiroz VAO, Pereira-Santos M, Santana MLP. Overweight and obesity associated with higher depression prevalence in adults: a systematic review and meta-analysis. J Am Coll Nutr. 2017 Mar-Apr;36(3):223-33.

6 Gariepy G, Nitka D, Schmitz N. The association between obesity and anxiety disorders in the population: a systematic review and metaanalysis. Int J Obes. 2010;34(3):407-19.

7 Amiri S, Behnezhad S. Obesity and anxiety symptoms: a systematic review and metaanalysis. Neuropsychiatr. 2019;33(2):72-89.

8 Specker S, de Zwaan M, Raymond N, Mitchell J. Psychopathology in subgroups of obese women with and without binge eating disorder. Compr Psychiatry. 1994 May-Jun;35(3):185-90.

9 Cleator J, Abbott J, Judd P, Sutton C, Wilding JP. Night eating syndrome: implications for severe obesity. Nutr Diabetes. 2012;2:e44.

10 Fabricatore AN, Wadden TA, Higginbotham AJ, Faulconbridge LF, Nguyen AM, Heymsfield $\mathrm{SB}$, et al. Intentional weight loss and changes in symptoms of depression: a systematic review and meta-analysis. Int J Obes. 2011;35(11):1363-76.

11 Brandheim S, Rantakeisu U, Starrin B. BMI and psychological distress in 68,000 Swedish adults: a weak association when controlling for an age-gender combination. BMC Public Health. 2013;13:68.

12 De Luca M, Angrisani L, Himpens J, Busetto L, Scopinaro N, Weiner R, et al. Indications for surgery for obesity and weight-related diseases: position statements from the international federation for the surgery of obesity and metabolic disorders (IFSO). Obes Surg. 2016;26(8):1659-96.
13 Lagerros YT, Rössner S. Obesity management: what brings success? Therap Adv Gastroenterol. 2013;6(1):77-88.

14 Jensen MD, Ryan DH, Apovian CM, Ard JD, Comuzzie AG, Donato KA, et al. 2013 AHA/ ACC/TOS guideline for the management of overweight and obesity in adults: a report of the American College of Cardiology/American Heart Association Task Force on Practice Guidelines and The Obesity Society. Circulation. 2014;129(25 Suppl 2):S102-38.

15 Hassan Y, Head V, Jacob D, Bachmann MO, Diu S, Ford J. Lifestyle interventions for weight loss in adults with severe obesity: a systematic review. Clin Obes. 2016;6(6):395-403.

16 Bauer K, Lau T, Schwille-Kiuntke J, Schild S, Hauner $\mathrm{H}$, Stengel A, et al. Conventional weight loss interventions across the different BMI obesity classes: a systematic review and quantitative comparative analysis. Eur Eat Disord Rev. 2020;28(5):492-512.

17 Montesi L, El Ghoch M, Brodosi L, Calugi S, Marchesini G, Dalle Grave R. Long-term weight loss maintenance for obesity: a multidisciplinary approach. Diabetes Metab Syndr Obes. 2016;9:37-46.

18 Corcelles R, Boules M, Froylich D, Hag A, Daigle CR, Aminian A, et al. Total weight loss as the outcome measure of choice after Rouxen-Y gastric bypass. Obes Surg. 2016;26(8): 1794-8.

19 Wolfe BM, Kvach E, Eckel RH. Treatment of obesity: weight loss and bariatric surgery. Circ Res. 2016;118(11):1844-55.

20 Lasikiewicz N, Myrissa K, Hoyland A, Lawton CL. Psychological benefits of weight loss following behavioural and/or dietary weight loss interventions. A systematic research review. Appetite. 2014;72:123-37.

21 Dawes AJ, Maggard-Gibbons M, Maher AR, Booth MJ, Miake-Lye I, Beroes JM, et al. Mental health conditions among patients seeking and undergoing bariatric surgery: a metaanalysis. JAMA. 2016;315(2):150-63.

22 Mack I, Ölschläger S, Sauer H, von Feilitzsch M, Weimer K, Junne F, et al. Does laparoscopic sleeve gastrectomy improve depression, stress and eating behaviour? A 4-Year Followup Study. Obes Surg. 2016;26(12):2967-73.

23 Wild B, Hünnemeyer K, Sauer H, Hain B, Mack I, Schellberg D, et al. A 1-year videoconferencing-based psychoeducational group intervention following bariatric surgery: results of a randomized controlled study. Surg Obes Relat Dis. 2015 Nov-Dec;11(6):1349-60.

24 Johnston BC, Kanters S, Bandayrel K, Wu P, Naji F, Siemieniuk RA, et al. Comparison of weight loss among named diet programs in overweight and obese adults: a meta-analysis. JAMA. 2014;312(9):923-33.

25 Gudzune KA, Doshi RS, Mehta AK, Chaudhry ZW, Jacobs DK, Vakil RM, et al. Efficacy of commercial weight-loss programs: an updated systematic review. Ann Intern Med. 2015; 162(7):501-12.

26 Dalle Grave R, Calugi S, Gavasso I, El Ghoch $M$, Marchesini G. A randomized trial of energy-restricted high-protein versus high-carbohydrate, low-fat diet in morbid obesity. Obesity. 2013;21(9):1774-81.

27 Goodpaster BH, Delany JP, Otto AD, Kuller L, Vockley J, South-Paul JE, et al. Effects of diet and physical activity interventions on weight loss and cardiometabolic risk factors in severely obese adults: a randomized trial. JAMA. 2010;304(16):1795-802.

28 Yumuk V, Tsigos C, Fried M, Schindler K, Busetto L, Micic D, et al. European guidelines for obesity management in adults. Obes Facts. 2015;8(6):402-24.

29 Bluher M. [Conservative obesity treatment: when and how?]. Dtsch Med Wochenschr. 2015;140(1):24-8.

30 Ahnis A, Figura A, Hofmann T, Stengel A, Elbelt U, Klapp BF. Surgically and conservatively treated obese patients differ in psychological factors, regardless of body mass index or obesity-related co-morbidities: a comparison between groups and an analysis of predictors. PLoS One. 2015;10(2):e0117460.

31 Kvalem IL, Bergh I, von Soest T, Rosenvinge JH, Johnsen TA, Martinsen EW, et al. A comparison of behavioral and psychological characteristics of patients opting for surgical and conservative treatment for morbid obesity. BMC Obes. 2015;3:6.

32 Fischer L, Wekerle AL, Sander J, Nickel F, Billeter AT, Zech U, et al. Is there a reason why obese patients choose either conservative treatment or surgery? Obes Surg. 2017;27(7):1684-90.

33 DGAV.S3-Leitlinie: Chirurgie der Adipositas und metabolischer Erkrankungen [2021 May 12]. Available from: https://www.awmf.org/ uploads/tx_szleitlinien/088-0011_S3_Chirurgie-Adipositas-metabolische-Erkrankugen_2018-02.pdf. 
34 Junne F, Ehehalt S, Ziser K, Reinehr T, Wiegand S, Mander J, et al. Targeting parental motivation for change in childhood obesity: development and validation of the PURICA-S scale. Int J Obes. 2019;43(11):2291-301.

35 Schmidt U, Magill N, Renwick B, Keyes A, Kenyon M, Dejong H, et al. The Maudsley Outpatient Study of treatments for anorexia nervosa and related conditions (MOSAIC): comparison of the Maudsley model of anorexia nervosa treatment for adults (MANTRA) with specialist supportive clinical management (SSCM) in outpatients with broadly defined anorexia nervosa: a randomized controlled trial. J Consult Clin Psychol. 2015; 83(4):796-807.

36 Wirth A, Wabitsch M, Hauner H. The prevention and treatment of obesity. Dtsch Arztebl Int. 2014;111(42):705-13.

37 Kroenke K, Spitzer RL, Williams JB. The PHQ-9: validity of a brief depression severity measure. J Gen Intern Med. 2001;16(9):60613.

38 Spitzer RL, Kroenke K, Williams JB, Löwe B. A brief measure for assessing generalized anxiety disorder: the GAD-7. Arch Intern Med. 2006;166(10):1092-7.

39 Ware JE, Kosinski M, Keller SD; New England Medical Center Hospital Health Institute. SF12: how to score the SF-12 physical and mental health summary scales. 3rd ed. 1998. p. 97.

40 Pudel V, Westhöfer J. Fragebogen zum Eßverhalten (FEV). Göttingen: Verl. f. Psychologie Dr. Hogrefe; 1989.

41 Alizai PH, Akkerman MK, Kaemmer D, Ulmer F, Klink CD, Ernst S, et al. Presurgical assessment of bariatric patients with the $\mathrm{Pa}-$ tient Health Questionnaire (PHQ): a screening of the prevalence of psychosocial comorbidity. Health Qual Life Outcomes. 2015;13: 80.

42 Batsis JA, Lopez-Jimenez F, Collazo-Clavell ML, Clark MM, Somers VK, Sarr MG. Quality of life after bariatric surgery: a populationbased cohort study. Am J Med. 2009;122(11): 1055.e1-10.
43 Wee CC, Davis RB, Hamel MB. Comparing the SF-12 and SF-36 health status questionnaires in patients with and without obesity. Health Qual Life Outcomes. 2008;6:11.

44 Bryant EJ, Rehman J, Pepper LB, Walters ER. Obesity and eating disturbance: the role of TFEQ restraint and disinhibition. Curr Obes Rep. 2019;8(4):363-72.

45 Loffler A, Luck T, Then FS, Sikorski C, Kovacs $\mathrm{P}$, Bottcher Y, et al. Eating Behaviour in the general population: an analysis of the factor structure of the German version of the threefactor-eating-questionnaire (TFEQ) and its association with the body mass index. PLoS One. 2015;10(7):e0133977.

46 IBM Corp. IBM SPSS Statistics for Windows. 24.0. Armonk, NY: IBM Corp.Released; 2016.

47 Conover WJ. Practical nonparametric statistics. New York: Wiley; 1971. Vol. 10; p.462.

48 Toussaint A, Hüsing P, Gumz A, Wingenfeld K, Härter M, Schramm E, et al. Sensitivity to change and minimal clinically important difference of the 7-item generalized anxiety disorder questionnaire (GAD-7). J Affect Disord. 2020;265:395-401.

49 Lubetkin EI, Gold MR. Areas of decrement in health-related quality of life (HRQL): comparing the SF-12, EQ-5D, and HUI 3. Qual Life Res. 2003;12(8):1059-67.

50 Little RJA, Rubin DB. Statistical analysis with missing data. New York: Wiley; 1987. Vol. 14; p. S278.

51 Lachin JM. Fallacies of last observation carried forward analyses. Clin Trials. 2016;13(2): 161-8.

52 Eekhout I, de Vet HC, Twisk JW, Brand JP, de Boer MR, Heymans MW. Missing data in a multi-item instrument were best handled by multiple imputation at the item score level. J Clin Epidemiol. 2014;67(3):335-42.

53 Cohen J. Statistical power analysis for the behavioral sciences. 1988; 15:415.
54 Huisman M, Kunst AE, Mackenbach JP. Inequalities in the prevalence of smoking in the European Union: comparing education and income. Prev Med. 2005;40(6):756-64.

55 Rudolph A, Hellbardt M, Baldofski S, de Zwaan M, Hilbert A. [Evaluation of the oneyear multimodal weight loss program DOC WEIGHT(R) 1.0 for obesity class II and III]. Psychother Psychosom Med Psychol. 2016; 66(8):316-23.

56 Teixeira PJ, Going SB, Sardinha LB, Lohman TG. A review of psychosocial pre-treatment predictors of weight control. Obes Rev. 2005; 6(1):43-65.

57 Williams GC, Grow VM, Freedman ZR, Ryan RM, Deci EL. Motivational predictors of weight loss and weight-loss maintenance. J Pers Soc Psychol. 1996;70(1):115-26.

58 Keranen AM, Savolainen MJ, Reponen AH, Kujari ML, Lindeman SM, Bloigu RS, et al. The effect of eating behavior on weight loss and maintenance during a lifestyle intervention. Prev Med. 2009;49(1):32-8.

59 Freund AM, Hennecke M. Changing eating behaviour vs. losing weight: the role of goal focus for weight loss in overweight women. Psychol Health. 2012;27(Suppl 2):25-42.

60 Kolotkin RL, Crosby RD, Williams GR, Hartley GG, Nicol S. The relationship between health-related quality of life and weight loss. Obes Res. 2001;9(9):564-71.

61 Kalarchian MA, Marcus MD, Courcoulas AP, Cheng Y, Levine MD. Preoperative lifestyle intervention in bariatric surgery: initial results from a randomized, controlled trial. Obesity. 2013;21(2):254-60.

62 Stewart F, Avenell A. Behavioural interventions for severe obesity before and/or after bariatric surgery: a systematic review and meta-analysis. Obes Surg. 2016;26(6):1203-14.

63 Nackers LM, Dubyak PJ, Lu X, Anton SD, Dutton GR, Perri MG. Group dynamics are associated with weight loss in the behavioral treatment of obesity. Obesity. 2015;23(8):1563-9. 